\title{
ANALISIS PARA EL DISEÑO DE LAS PALAS DE UNA TURBINA MARINA
}

\author{
I. Olivares, M. Santos \\ Universidad Complutense de Madrid, España \\ irene.0sm@gmail.com,msantos@ucm.es \\ M. Tomás-Rodríguez \\ City University of London, UK, \\ maria.tomas-rodriguez.1@city.ac.uk
}

\begin{abstract}
Resumen
En este trabajo se hace un análisis de algunos efectos que tienen lugar en las palas del rotor de una turbina marina y se estudia su influencia sobre la eficiencia de las mismas. Esto permite seleccionar un tipo de perfil de las palas y diseñar algunas de sus características para optimizar la producción de energía. En concreto se ha hecho un análisis para distintos ángulos de ataque, obteniendo conclusiones muy interesantes para la eficiencia y condiciones de trabajo de estas turbinas.
\end{abstract}

Palabras Clave: Turbinas marinas, energía marina, palas, energía renovable.

\section{INTRODUCCIÓN}

La creciente necesidad de generación energética, unida a la inestabilidad de precios y efectos perniciosos sobre el medio ambiente asociados al petróleo y otras fuentes de energía basadas en combustibles fósiles, ha renovado el interés por la búsqueda de nuevas fuentes renovables [10]. Entre ellas, uno de los recursos energéticos menos explotados es el que puede generarse a partir de las corrientes marinas y de las mareas [2].

El movimiento de las masas de agua debida a las mareas tiene su origen en la posición relativa entre la Tierra, el Sol y la Luna, que inducen un movimiento periódico y predecible. La predictibilidad de este recurso es una característica de gran importancia ya que facilita la planificación de la distribución energética, en contraste a otras fuentes renovables como la energía solar o eólica [7].

Por otra parte, la tecnología necesaria para su extracción presenta la ventaja de estar parcialmente desarrollada gracias a su similitud con las turbinas eólicas, pudiendo traspasar parte del conocimiento de un campo a otro.
Otra ventaja es que la densidad del agua del mar es aproximadamente 800 veces mayor que la del aire, haciendo posible la disminución de las dimensiones de las turbinas para la extracción de una misma potencia.

Algunos de los mayores retos para el desarrollo de las turbinas de mar son los altos costes de instalación y mantenimiento que conlleva la operación en zonas de difícil acceso, la necesidad de estructuras robustas capaces de soportar las cargas que genera un fluido de alta densidad en comparación con el aire, así como el efecto de cavitación, que afecta principalmente a la zona de punta de pala del rotor causando daños que provocan la disminución de la eficiencia de la turbina [11].

Teniendo en cuenta estas necesidades, en este trabajo se desarrolla la optimización del diseño de las palas de una turbina de mar de eje horizontal para aprovechar de forma más eficiente este recurso.

El artículo está estructurado como sigue. En la siguiente sección se presenta de forma breve $\mathrm{y}$ simplificada el estudio hidrodinámico de las palas de una turbina marina. En la sección 3 se analiza el perfil de las palas, seleccionando una específica. La influencia de algunos coeficientes se muestra en la sección 4. El trabajo termina con las conclusiones y los trabajos futuros.

\section{ESTUDIO HIDRODINÁMICO DE LAS PALAS}

Partiendo de modelos del recurso marino, tanto de las mareas como de las corrientes, previamente desarrollados [9], se va a analizar el perfil hidrodinámico de las palas de una turbina.

De esta forma se podrá diseñar dicho perfil de las palas del rotor de forma que se optimice la energía que se puede obtener mediante una turbina marina, ya que la eficiencia de operación de una turbina vendrá determinada en gran medida por las características de las palas del rotor utilizado [1]. 
En el caso de las turbinas de mar, los perfiles más comunes son similares a aquellos usados por turbinas eólicas. Sin embargo, a la hora de la elección y diseño del perfil, es necesario tener en cuenta ciertas particularidades que atañen especialmente a este tipo de turbinas [8].

Entre ellos cabe destacar, además del efecto de cavitación, la necesidad de perfiles robustos debido a que las cargas que sufrirá la estructura aumentan debido a la mayor densidad del fluido de trabajo.

\subsection{FUERZAS DE SUSTENTACION Y ARRASTRE}

El flujo de agua sobre el perfil de la pala producirá una distribución de fuerzas alrededor de ésta. La velocidad de dicho flujo aumentará sobre la zona convexa resultando en una disminución de la presión en esa superficie de la pala con respecto a la superficie cóncava. Como resultado de ese gradiente de presiones aparecerá una fuerza de sustentación que "tirará" de la pala en dirección perpendicular al flujo.

Por otra parte, el efecto de rozamiento viscoso frenará las partículas del fluido en contacto con la superficie, creando un gradiente de velocidades en la zona cercana a ésta. Como resultado aparecerá una fuerza neta de arrastre con igual dirección que el flujo.

Para el análisis de los problemas hidrodinámicos es útil la definición de ciertos parámetros adimensionales relacionados con las anteriores fuerzas [4].

El primero de ellos es el número de Reynolds, que define las características del flujo de trabajo.

$$
R e=\frac{\rho v L}{\mu}
$$

donde $\rho, v, \mu$ son respectivamente, la densidad, la velocidad y la viscosidad dinámica del fluido; L es la longitud que caracteriza el objeto que se está analizando.

En el caso de las turbinas de mar, la densidad del agua del mar es de alrededor de $1025 \mathrm{~kg} / \mathrm{m}^{3}$, las velocidades de las corrientes se encuentran típicamente entre $1 \mathrm{~m} / \mathrm{s}$ y $2 \mathrm{~m} / \mathrm{s}$ de media, y la viscosidad dinámica es de $0.001139 \mathrm{~m}^{2} / \mathrm{s}$ a una temperatura de $15^{\circ} \mathrm{C}$.

Si suponemos una turbina de $10 \mathrm{~m}$ de radio, obtenemos un número de Reynolds aproximado de $\mathrm{Re}=9 \cdot 10^{6}$ (a velocidad media de $\left.1 \mathrm{~m} / \mathrm{s}\right)$.

Este parámetro tendrá un fuerte efecto en las fuerzas resultantes ya que según decrece el número de Reynolds, las fuerzas viscosas aumentan en magnitud con respecto a las fuerzas inerciales del fluido, afectando a la velocidad del mismo, a su gradiente de presiones $\mathrm{y}$, por último, a la fuerza de sustentación. Podemos definir dos coeficientes adimensionales que cuantifican la magnitud de las fuerzas de sustentación $\mathrm{y}$ arrastre y que dependerán tanto del número de Reynolds como del ángulo de ataque:

$$
\begin{aligned}
& C_{l}=\frac{F . \text { sustentación/unidad longitud de pala }}{\text { F.dinámica del fluido/unidad longitud de la pala }} \\
& C_{D}=\frac{\text { F. arrastre/unidad longitud de pala }}{\text { F.dinámica del fluido/unidad longitud de la pala }}
\end{aligned}
$$

Para optimizar las características de la turbina será necesario maximizar el coeficiente de sustentación $(C l)$ y minimizar el de arrastre $\left(C_{d}\right)$.

\subsection{COEFICIENTE DE PRESION}

Otro de los coeficientes importantes para el análisis de perfiles hidrodinámicos, y en concreto para las turbinas de mar, es el coeficiente de presión, definido como:

$$
C_{p}=\frac{P_{L}-P_{0}}{\frac{1}{2} \rho v_{\text {fluid }}^{2}}
$$

donde $P_{L}$ es la distribución local de presiones a lo largo de la pala, $P_{0}$ es la presión del flujo libre y $v_{\text {fluid }}$ es la velocidad local del fluido.

Cuanto mayor (menos negativo) sea este coeficiente, menor diferencia habrá entre la presión local en la superficie de la pala con respecto a la presión del flujo libre.

El fenómeno de cavitación, mencionado anteriormente, sucede cuando la presión local en un punto de la pala cae por debajo de la presión de vapor del fluido.

Es decir, cuando el coeficiente de presión toma el valor:

$$
C_{p}=\frac{P_{V}-P_{0}}{\frac{1}{2} \rho v_{\text {fluid }}^{2}}=-K_{f}
$$

donde se ha definido el "coeficiente de cavitación", $K_{f}$, que da cuenta de la magnitud de este fenómeno.

Cuando se produce este efecto, las moléculas que componen el fluido pasen a estado de vapor, formándose burbujas que viajan a zonas de mayor presión e "implosionan" regresando a su forma líquida de forma súbita, produciendo el deterioro de la superficie de la pala, con fuertes efectos sobre el coeficiente de sustentación y de arrastre. 
Por tanto, es de gran importancia el análisis del perfil, así como de las condiciones de trabajo de la turbina para la minimización de estos efectos.

\section{ANALISIS DEL PERFIL DE LAS PALAS}

El análisis del perfil se puede llevar a cabo mediante el estudio de la distribución del coeficiente de presión.

Es necesario que este coeficiente se encuentre por encima de un valor mínimo que variará según el rango de velocidades de corriente incidentes, debiéndose cumplir en todo momento que $P_{L}>P_{V}$ y que $-C_{p}>K_{f}$.

Cuanto más positivo sea $\mathrm{Cp}$, mayor será la presión local; sin embargo, menor será a su vez el coeficiente de sustentación, ya que esta fuerza se origina directamente del gradiente de presiones.

Se elegirá aquel perfil que aumente $\mathrm{C}_{\mathrm{p}}$ siempre intentando mantener $\mathrm{C}_{1}$ en valores lo más altos posibles. En concreto, uno de los datos más significativos viene dado por la ratio $\mathrm{C}_{\mathrm{l}} / \mathrm{C}_{\mathrm{d}}$, con el que se obtiene una comparativa entre la magnitud de las fuerzas de sustentación y de arrastre.

Dicho parámetro tiene un efecto determinante en el coeficiente de potencia de la turbina, tal y como se puede observar en la figura 1.

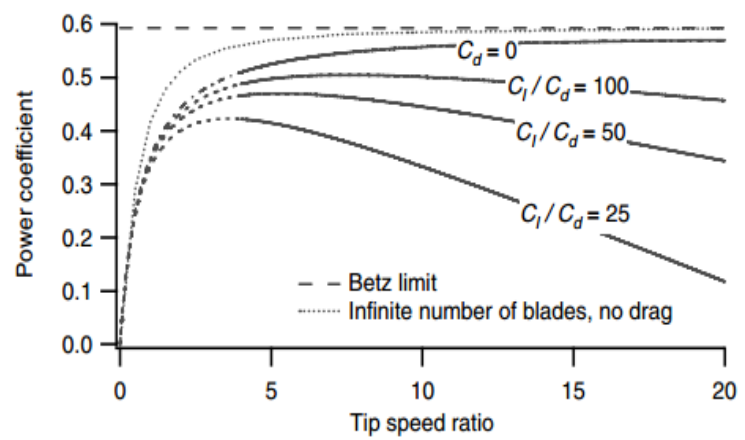

Figura 1: Coeficiente de potencia en función de la velocidad especifica (TSR, Tip Speed Ratio) para distintos coeficientes de sustentación y arrastre

\subsection{CARACTERISTICAS DE LOS PERFILES}

Para la realización de este análisis se han escogido cuatro perfiles comúnmente utilizados para las turbinas de mar, así como para la fabricación de aerogeneradores $[3,5]$.

Los perfiles de pala utilizados en turbinas eólicas tienen su origen en aquellos utilizados en la aviación. Típicamente se agrupan en familias estandarizadas cuyo nombre es identificativo de ciertos parámetros característicos del perfil [6].
En concreto, los perfiles que se presentan en este trabajo son: NACA 63415, NACA 64415, NACA 65415 y NACA 63815.

La Figura 2 servirá para explicar el significado de cada uno de los dígitos de los anteriores perfiles:

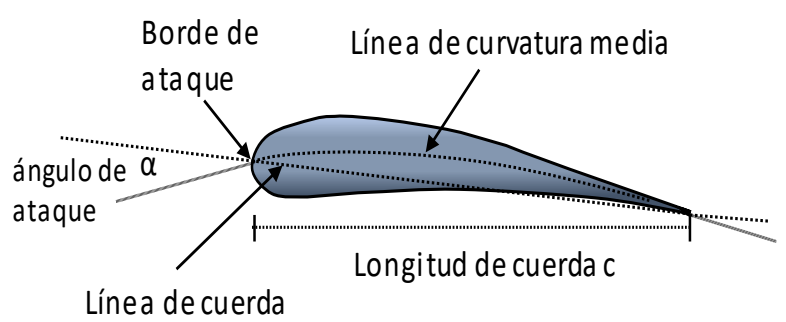

Figura 2: Parámetros que caracterizan el perfil hidrodinámico de una pala

- Linea de curvatura media - línea que recorre la pala en su longitud en el punto medio de su anchura.

- Línea de cuerda - línea que une el borde de ataque y el borde de fuga del perfil.

- Curvatura máxima - Distancia máxima entre la línea de cuerda y la línea de curvatura media.

- Espesor máximo - Distancia máxima entre la superficie superior e inferior.

- Radio del borde de ataque - Medida del afilamiento del borde de ataque. Puede variar desde un $0 \%$ para perfiles afílados supersónicos a un $2 \%$ de la longitud de cuerda (c) para perfiles achatados.

En los perfiles NACA de 5 dígitos, la primera cifra es proporcional al coeficiente de sustentación del perfil; los dos siguientes indican el doble de la posición de curvatura máxima y las dos últimas indican el espesor máximo, ambos en porcentaje de cuerda.

En la Figura 3 se presentan los perfiles de estudio y una tabla adjunta en la que se especifican los coeficientes de sustentación y arrastre para un ángulo de ataque de $0^{\circ}$.

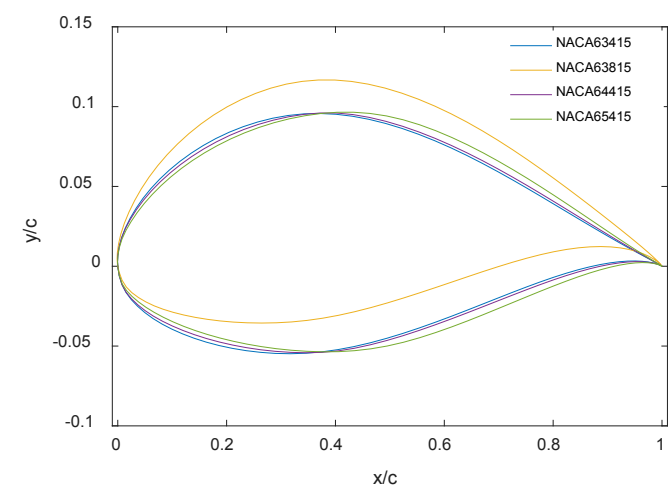

Figura 3: Perfil de las palas de estudio

Los valores de los coeficientes obtenidos para cada perfil se muestran en la tabla 1, donde se puede 
observar la gran diferencia que existe entre los valores de $C_{L}$ y $C_{L} / C_{D}$ del perfil NACA 63815 con respecto al resto.

Tabla 1: Coeficientes de sustentación y arrastre y su ratio para los perfiles de estudio (NACA)

\begin{tabular}{|l|lll|}
\hline Perfil & $\mathrm{C}_{\mathrm{L}}$ & $\mathrm{C}_{\mathrm{D}}$ & $\mathrm{C}_{\mathrm{L}} / \mathrm{C}_{\mathrm{D}}$ \\
\hline $\mathbf{6 3 4 1 5}$ & 0,433 & 0,01393 & 30.65 \\
$\mathbf{6 3 8 1 5}$ & 0,847 & 0,01445 & 58.61 \\
$\mathbf{6 4 4 1 5}$ & 0,430 & 0,01403 & 30.22 \\
$\mathbf{6 5 4 1 5}$ & 0,430 & 0,01423 & 31.08 \\
\hline
\end{tabular}

En la Figura 4 se muestra la distribución del coeficiente de presión de los distintos perfiles para un ángulo de $0^{\circ}$.

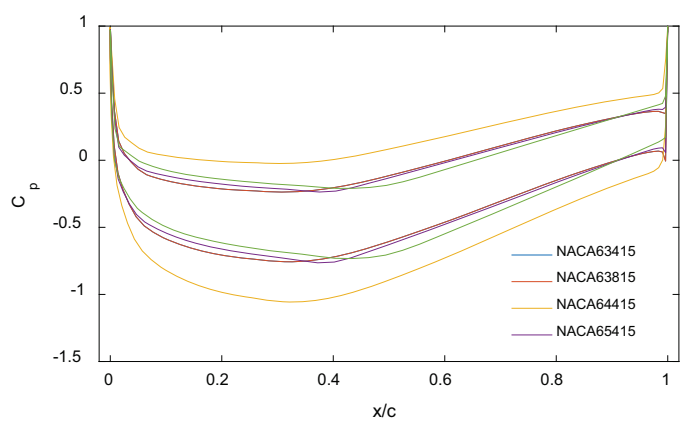

Figura 4: Distribución del coeficiente de presión para los perfiles de estudio

En ella se observa que el perfil con mejor ratio $C_{L} / C_{D}$ (NACA63815) es también aquel con menor coeficiente de presión, lo que indica la aparición de cavitación a velocidades más bajas limitando el rango de velocidades de operación de la turbina en mayor medida.

A pesar de ello, se elegirá este perfil por su alto coeficiente de sustentación y se estudiará las condiciones adecuadas de operación de la turbina para evitar la aparición de este efecto.

Para realizar el estudio se ha considerado una turbina de radio $\mathrm{R}=10 \mathrm{~m}$ y una profundidad de inmersión de $10 \mathrm{~m}$ respecto al punto más alto que alcanzan las palas del rotor. Así mismo, se han considerado valores típicos para la presión de vapor del agua $\mathrm{P}_{\text {vapor }}=2000$ $\mathrm{Pa}$ y para la presión atmosférica de $\mathrm{P}_{\mathrm{AT}}=101320 \mathrm{~Pa}$. Para el análisis de las condiciones de operación se ha supuesto una velocidad de rotación de $\Omega=11.5 \mathrm{rpm}$.

\section{INFLUENCIA DE LOS COEFICIENTES}

Una vez seleccionado el perfil, es posible incrementar el coeficiente de sustentación variando el ángulo de ataque a valores mayores que cero, sufriendo un descenso a partir de $\operatorname{los} 10^{\circ}$, tal y como se observa en la figura 5.

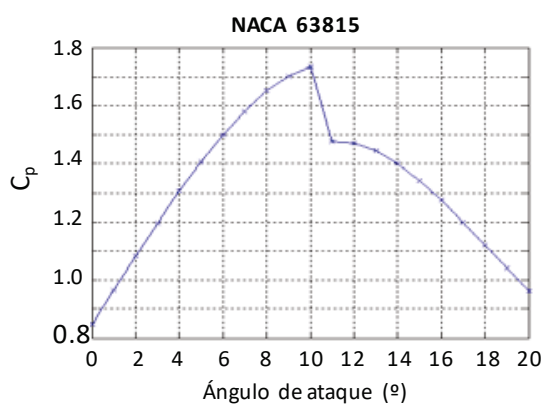

Figura 5: Coeficiente de sustentación en función del ángulo de ataque

Sin embargo, si se analizan las distribuciones de velocidades y del coeficiente de presión que aparecen en las siguientes imágenes para ángulos desde $0^{\circ}$ hasta $10^{\circ}$, podemos ver que éste último alcanza valores mínimos de $\mathrm{C}_{\mathrm{p}}=-7$. Como se puede observar en la figura 6 , mientras que la velocidad del fluido aumenta hasta casi tres veces en una de las caras de la pala, en la opuesta alcanza prácticamente valores nulos cerca del borde de ésta. En esa zona, la presión bajará fuertemente con riesgo de pasar a estado vapor y provocar daños en la estructura de la turbina.

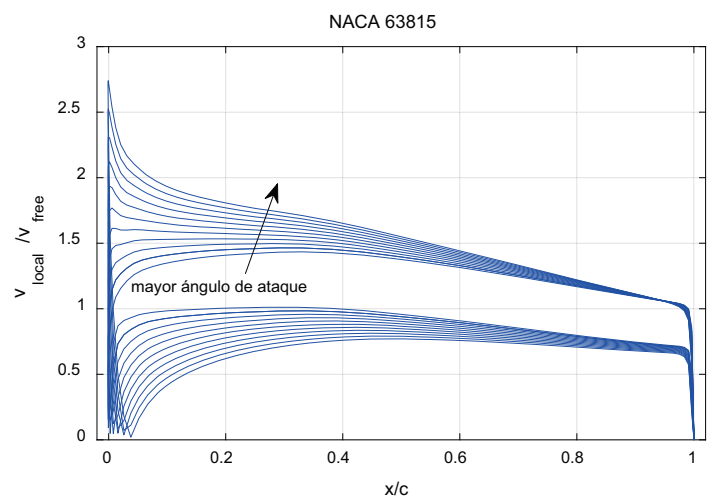

NACA 63815

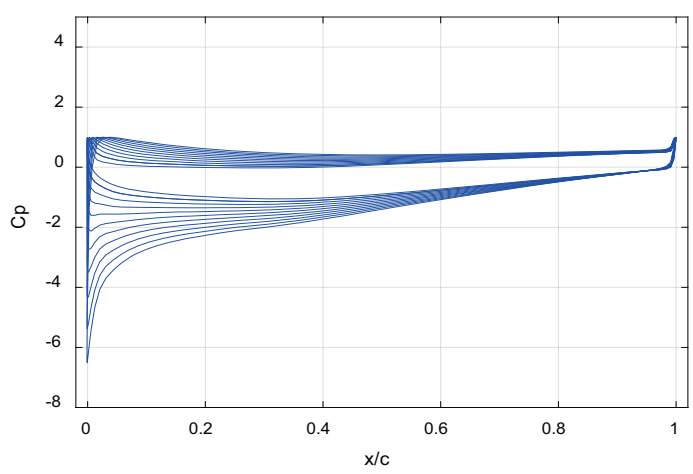

Figura 6: Distribución de velocidades (arriba) y del coeficiente de presión (abajo) a lo largo del perfil de pala

Utilizando la ecuación (5) podemos calcular el coeficiente de cavitación a lo largo de las palas de la turbina en operación. Suponiendo una velocidad de 
corriente típica de $2 \mathrm{~m} / \mathrm{s}$, que se puede observar en los picos de corrientes de datos reales, la velocidad local del fluido en cada punto de la longitud de la pala se ha obtenido como:

$$
v_{\text {fluid }}=\sqrt{v_{\text {free }}^{2}(1-a)^{2}+\Omega^{2} r^{2}\left(1-a^{\prime}\right)}
$$

donde a y a' son, respectivamente, el factor axial y tangencial de inducción. El factor axial viene definido como $a=\left(1-\frac{v_{\text {local }}}{v_{\text {free }}}\right)$, mientras que para el factor tangencial se ha aproximado $\mathrm{a}=0$ ya que suele tomar valores muy bajos en la zona de interés (zonas cercanas a la punta de pala).

En la Figura 7 se muestra el coeficiente de cavitación a lo largo de la pala, donde se pueden observar valores mínimos de alrededor de $\mathrm{K}_{\mathrm{f}}=4$.

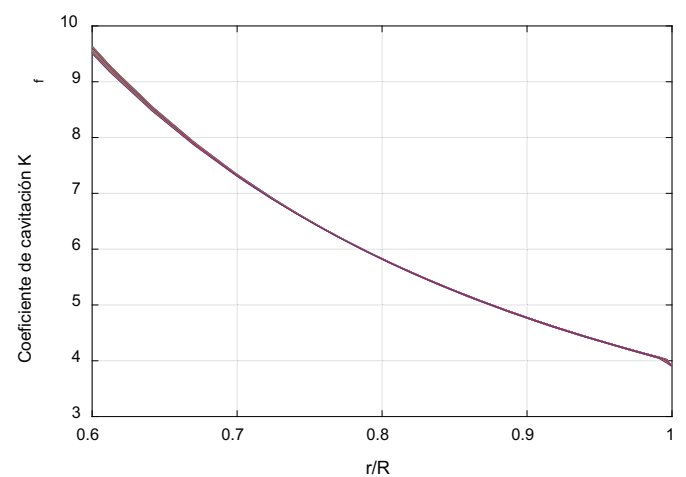

Figura 7: Coeficiente de cavitación para cada punto de la pala.

Para estas condiciones, por tanto, será posible la aparición del efecto de cavitación, ya que se debería cumplir en todo momento que $\mathrm{K}_{\mathrm{f}}>-\mathrm{C}_{\mathrm{p}} \mathrm{y}$, sin embargo, el coeficiente de presión toma valores por debajo de 4 para las áreas cercanas los bordes del perfil de pala.

Para evitar este efecto, será necesario mantener un ángulo de ataque por debajo de un ángulo máximo que asegure unas condiciones de trabajo adecuadas.

El ángulo máximo de trabajo, en este caso, sería de alrededor de $6^{\circ}$. Utilizando el perfil NACA 63815 se obtienen los valores de los coeficientes de sustentación, arrastre y presión, así como la distribución de velocidades y presiones mostrados en las figuras 8 y Tabla 2.

Tabla 2: Coeficientes de sustentación, arrastre y su ratio para un ángulo de ataque de $6^{\circ}$

\begin{tabular}{|l|llll|}
\hline Perfil & $\boldsymbol{\alpha}\left(^{\boldsymbol{0}}\right)$ & $\mathbf{C L}$ & $\mathbf{C D}$ & $\mathbf{C L} / \mathbf{C D}$ \\
\hline $\mathbf{6 3 8 1 5}$ & 6 & 1,487 & 0,02203 & 67.6 \\
\hline
\end{tabular}
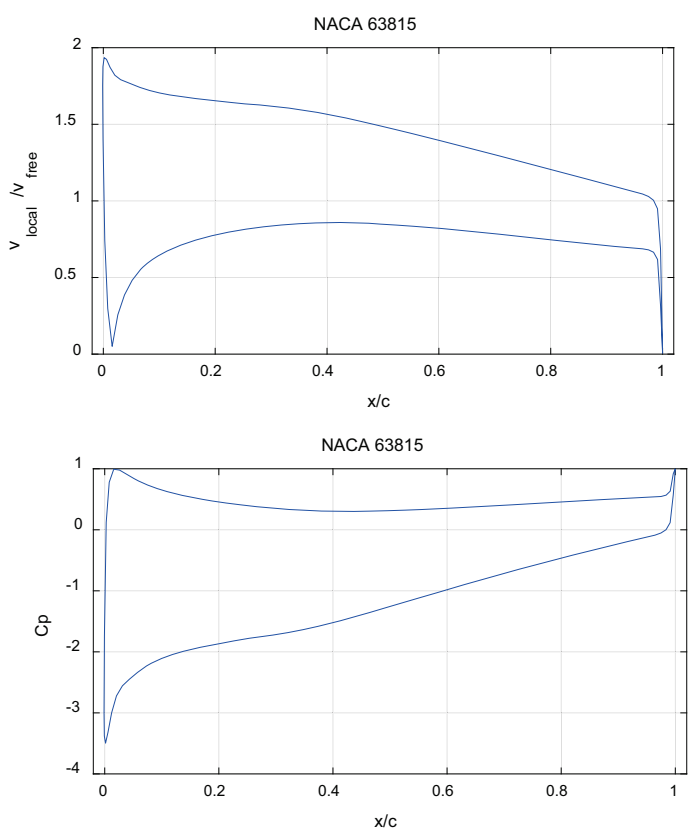

Figura 8: Distribución de la ratio de velocidades locales (arriba) y coeficiente de presión (abajo) para un ángulo de $6^{\circ}$

Suponiendo estos valores para la ratio $C_{L} / C_{D}$, es posible obtener el coeficiente de potencia de la turbina como:

$C_{p, \max }=\left(\frac{16}{27}\right) \lambda\left[\lambda+\frac{1.32+\left(\frac{\lambda-8}{20}\right)^{2}}{N^{2 / 3}}\right]^{-1}-\frac{0.57 \lambda^{2}}{\frac{C_{l}}{C_{d}}\left(\lambda+\frac{1}{2 N}\right)}$

donde se define $\lambda=\frac{\Omega R}{v_{\text {free }}}$ con $\Omega$ expresado en $\mathrm{rad} / \mathrm{s}$. Ecuación que se ajusta con un $0.5 \%$ de precisión para ratios de velocidad de punta de pala de entre 4 a 20 y para cocientes $C_{L} / C_{D}$ desde 25 a infinito. Con los datos anteriores se obtiene un coeficiente máximo de potencia de $C_{p, \max }=0.48$.

Esta ecuación, con los datos encontrados, será la que mejor define el comportamiento de la turbina y por lo tanto la que se implementaría en un modelo de turbina marina.

\section{CONCLUSIONES Y TRABAJOS FUTUROS}

En el presente trabajo se ha llevado a cabo un análisis del diseño de las palas del rotor de una turbina marina para optimizar la extracción de energía a partir de las mareas.

El estudio ha sido desarrollado teniendo en cuenta las características particulares de este tipo de dispositivos, como la dificultad de mantenimiento y la necesidad de una estructura que minimice el efecto de cavitación, 
fenómeno que deteriora de forma acusada las palas del rotor.

Tras el análisis para el perfil hidrodinámico de las palas, se ha elegido un perfil NACA 63815, que cumple con los requisitos especificados, obteniendo la influencia de los coeficientes sobre las mismas.

Como trabajos futuros se plantea la simulación de la turbina con estas palas, para obtener la producción de energía a partir de la predicción del recurso marino.

\section{English summary}

\section{ANALYSIS FOR THE DESIGN OF MARINE TURBINE BLADES}

\begin{abstract}
An analysis of the effects taking place in the rotor blades of a marine turbine is carried out in this work, studying their influence on its operating efficiency. Based on these results, it is possible to select and design the characteristics of the blades section to optimize the energy extraction. In addition, different attack angles have been analysed with interesting results on the efficiency and optimum operating conditions of the turbine.
\end{abstract}

Keywords: marine turbine, marine energy, blades, renewable energy.

\section{Referencias}

[1] Ahmed, M. R. (2012). Blade sections for wind turbine and tidal current turbine applicationscurrent status and future challenges. International Journal of Energy Research, 36(7), 829-844.

[2] Bahaj, A. S. (2011). Generating electricity from the oceans. Renewable and Sustainable Energy Reviews, 15(7), 3399-3416.

[3] Batten, W. M. J., Bahaj, A. S., Molland, A. F., Chaplin, J. R. (2006). Hydrodynamics of marine current turbines. Renewable energy, 31(2), 249256.

[4] Batten, W. M. J., Bahaj, A. S., Molland, A. F., \& Chaplin, J. R. (2008). The prediction of the hydrodynamic performance of marine current turbines. Renewable energy, 33(5), 1085-1096.
[5] González-Longatt, F. (2006, April). Turbina de Viento: Caracterización de Operación. In: CIBELEC 2005 II Congreso Iberoamericano de Estudiantes de Ingeniería Eléctrica, Electrónica y Computación.

[6] Jamieson, P. (2018). Innovation in wind turbine design. John Wiley \& Sons.

[7] Mikati, M., Santos, M., Armenta, C. (2012). Modelado y simulación de un sistema conjunto de energía solar y eólica para analizar su dependencia de la red eléctrica. Revista Iberoamericana de Automática e Informática industrial, 9(3), 267-281.

[8] Nicholls-Lee, R. F., Turnock, S. R., \& Boyd, S. W. (2008). Simulation based optimisation of marine current turbine blades, 314-328.

[9] Olivares I., Santos M. (2015) "Simulación y modelización de las mareas y análisis de su aprovechamiento por una turbina de mar", Actas XXXVI Jornadas de Automática, CEA-IFAC, 864-870.

[10] Romera-Cabrerizo, J.A, Santos, M. (2017) "ParaTrough v1.0: Librería en Modelica para simulación de plantas termosolares", Revista Iberoamericana de Automática e Informática Industrial, 14, 4, 412-423.

[11] Zhou, Z., Benbouzid, M., Charpentier, J. F., Scuiller, F., Tang, T. (2017). Developments in large marine current turbine technologies-A review. Renewable and Sustainable Energy Reviews, 71, 852-858.

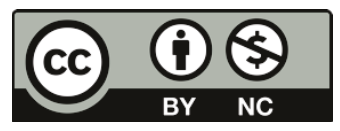

(C) 2018 by the authors. Submitted for possible open access

publication under the terms and conditions of the Creative 